\title{
Unusual Presentation of Choriocarcinoma Six Months after Term Delivery: A Case Report
}

\author{
Prerna Dhabarde ${ }^{1}$, Archana Joshi ${ }^{2}$, Sadhana Mahore ${ }^{3}$
}

\begin{abstract}
Choriocarcinoma coexisting with or after an otherwise normal pregnancy is extremely rare. Choriocarcinoma invades and metastasis early and is often widespread at the time of diagnosis. A 25 year old female complaining of bleeding p/v since 1 month with spontaneous expulsion of a mass per vaginum, after post partal amenorrhoea of 5 months. A history of contact was present. In this case, the diagnosis was established by histopathological examination of expelled tissue mass. Adequate tissue samples are necessary for histopathological diagnosis and early diagnosis of gestational choriocarcinoma can be done by routine histopathological examination of the placenta after birth.
\end{abstract}

Keywords: GTD: gestational trophoblastic disease, B-hCG: $\beta$-human chorionic gonadotropin, GCO: gestational choriocarcinoma, NGCO: non gestational choriocarcinoma

\section{Introduction}

Choriocarcinoma coexisting with or after an otherwise "normal" pregnancy is extremely rare, with an estimated occurrence of 1 per 160,000 pregnancies ${ }^{1}$. Choriocarcinoma is frequently preceded by a complete mole, ectopic pregnancy, nonmolar intrauterine abortion, and uncommonly by a partial mole

Gestational choriocarcinoma is a highly malignant tumor of trophoblastic cells with a propensity to metastasize to various sites ${ }^{2}$. Choriocarcinoma invades and metastasis early and is often widespread at the time of diagnosis. Presently signs and symptoms of gestational choiocarcinoma are highly variable in patients with gtd, gynecological symptoms are sometime ignored, attributed to normal peripartum or vaginal bleeding in puerperium or may be presented by non classic manifestation. ${ }^{3-4}$ the ultimate cause of gestational trophoblastic disease is claimed to be genetic in origin. No environmental etiological factor has been implicated up till now apart from the deficient vitamin-a precursor 'carotene' in diet $^{3 .}$ Most cases of the choriocarcinoma after term pregnancy were diagnosed only by histopathological examination $^{3}$. We report a case of a 25 year-old woman diagnosed with choriocarcinoma manifesting six months after a term pregnancy, presenting as retained products of conception.

\section{Case Report}

A 25 year old female complaining of bleeding $\mathrm{p} / \mathrm{v}$ since 1 month, after post partal amenorrhoea of 5 months. A history of contact was present. Spontaneously she expelled a mass per vaginum on $\mathrm{p} / \mathrm{s}$ examination, cervix and vagina were normal, bleeding was present $\mathrm{p} / \mathrm{v}$ and some products were felt in os. On $\mathrm{p} / \mathrm{v}$ examination uterus was 8 weeks. A provisional diagnosis of incomplete abortion with retained products of conception was made clinically. Dilatation and evacuation was done on the same day. An expelled mass along with evacuated products of conception were sent for histopathological examination.

Gross examination showed a single, irregular, greyishbrown, firm tissue mass which was measuring $3 \times 2 \times 1 \mathrm{~cm}$ along with multiple, irregular, brownish, soft, friable tissue bits total measuring about $3.5 \times 2.5 \times 1 \mathrm{~cm}$.
Microscopic examination from a single firm tissue mass revealed tumor composed of sheets of cytotrophoblast and syncytotrophoblast. Tumor cells showed marked pleomorphism,anisonucleosis, and mitotic figures. Chorionic villi not seen. Large areas of hemorrhage and necrosis also seen [fig.1 \& fig. 2]. Sections from irregular tissue bits revealed a foci of small endometrial glands and stroma also seen. Glands were lined by low columner cells.

Histopathologically the diagnosis of gestational choriocarcinoma was given. Subsequently $\beta$-hcg levels were done and found to be raised $(600000 \mathrm{iu} / \mathrm{ml})$.

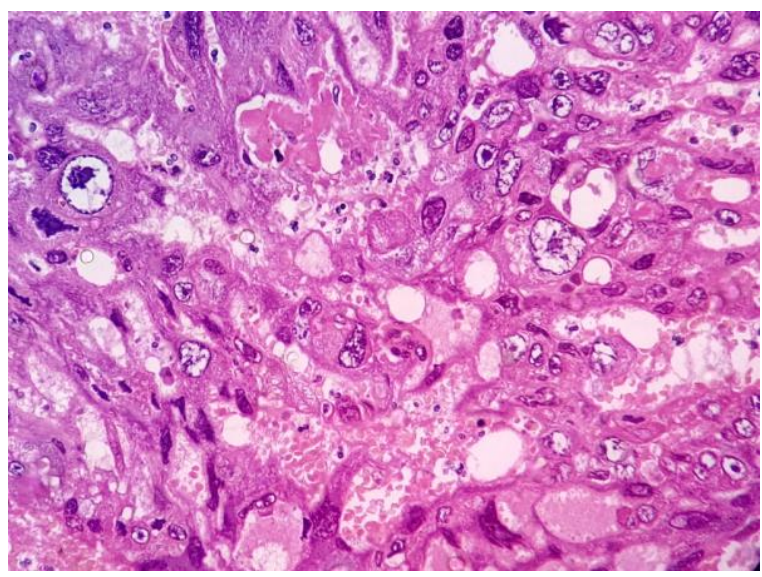

Figure 1

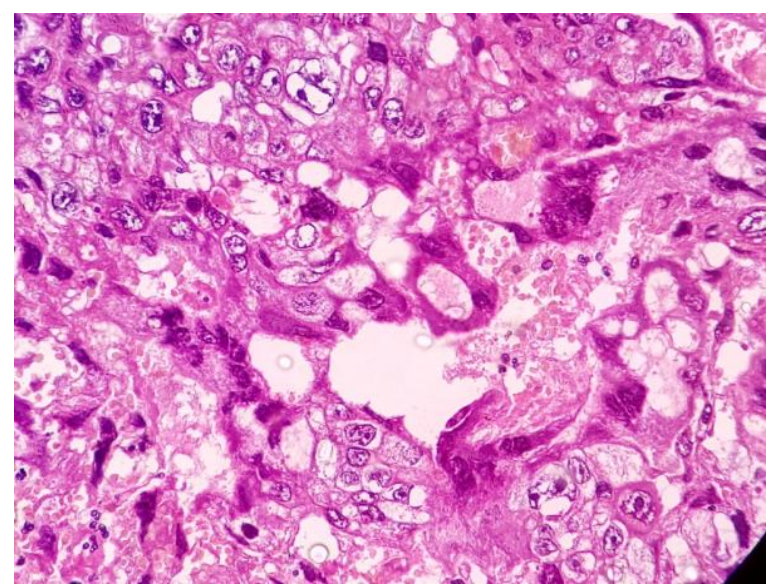

Figure 2

Volume 5 Issue 6, June 2016 www.ijsr.net 


\section{International Journal of Science and Research (IJSR) \\ ISSN (Online): 2319-7064 \\ Index Copernicus Value (2013): 6.14 | Impact Factor (2015): 6.391}

\section{Discussion}

Choriocarcinoma is a highly malignant trophpblastic tumor. It can arise from any type of trophoblastic tissue (molar pregnancy, abortion, ectopic pregnancy) but occurs very rarely after a normal term Pregnancy.

Choriocarcinoma is categorized as either gestational or nongestational. Gestational choriocarcinoma can occur following a pregnancy and is characterized by its presentation in the uterine corpus. On the other hand, nongestational choriocarcinoma is thought to arise from germ cells and is characterized by its presentation in males and children. The distinction between NGCO and GCO is important because of the worse prognosis of NGCO which requires more aggressive therapy with multiple chemotherapeutic agents. $^{3}$

Histologically, choriocarcinoma consists of sheets of anaplastic cytotrophoblast and syncytiotrophoblast tissue without chorionic villi. While some trophoblasts, which are intermediate in appearance, may also be seen, the biphasic pattern of obviously malignant appearing mononuclear (cytotrophoblast) and multinuclear cells (syncytiotrophoblast) is essentially pathognomonic of choriocarcinoma. Extensive necrosis, hemorrhage, and vascular invasion are common hence, extensive sampling is essential for histopathological diagnosis ${ }^{5}$.

Simple macroscopic examination of the placenta after delivery can identify suspicious placental areas. Macroscopically, choriocarcinoma appears like white nodules and infracted areas in the placental mass ${ }^{5}$. Gestational trophoblastic neoplasia after a normal term pregnancy is always choriocarcinoma and its early diagnosis is important ${ }^{5}$. Histopathological examination of the placenta after birth in all obstetric centers is not done routinely, causing delayed diagnosis of choriocarcinoma. Persistent elevation of serum $\beta$ hcg following any non-molar pregnancy, miscarriage, ectopic, or preterm/term pregnancy, may be due to development of choriocarcinoma. Serial testing of $\beta$ hcg serum concentrations is Essential in monitoring treatment and confirming remission. In this case, the diagnosis was established by histopathological examination of expelled tissue mass. It is necessary to have adequate tissue samples for histopathological diagnosis 5 . Delay of the diagnosis by the lack of histopathological examination affect the outcome of the case by increasing the risk of metastasis and increased resistance to monochemotherapy ${ }^{5}$ Anthony todd presented an unusual case of choriocarcinoma occurring 12 months after delivery. Therefore choriocarcinoma should be considered in women of childbearing age with acute haemorrhage occurring even years after a normal birth. ${ }^{6}$

Choriocarcinoma is a curable malignancy with a poor prognosis if not diagnosed early.

\section{Conclusion}

The presented case emphasizes the importance of the microscopic analysis of all placentas postpartum, because even if the incidence of gestational choriocarcinoma after a normal pregnancy is low, the prompt diagnosis significantly improves the patient's prognosis. It is our belief that the incidence of placental choriocarcinoma may actually be higher than reported. By reporting this case, we support the opinion of early diagnosis of gestational choriocarcinoma by routine histopathological examination of the placenta after birth. For the diagnosis of gestational choriocarcinoma the pathologist needs sufficient tumor sample due to large areas of bleeding and tumor necrosis specific to this tumor.

\section{References}

[1] Ganapathi, Karthik A. Paczos, Tamera ; George, Mary D; Goodloe, Samuel Balos, Lucia L.; Chen, Frank. (2010), International Journal Of Gynecological Pathology,.

[2] Mehmet Y., Ozcan Y., Cigdem P. And Nukhet T;(2009), Gestational Choriocarcinoma Diagnosed With Spontaneous Splenic Rupture After Pregnancy Induced By In Vitro Fertilization: A Case Report; Cases Journal, 2:7518.

[3] Berkowitz R S, Cramer Dw, Bernstein Mr, Cassells S, Driscoll Sg, Goldstein Dp:(1985) Risk Factors For Complete Molar Pregnancy From A Case-Control Study; American Journal Of Obstetrics And Gynecology, 152:1016-1020.

[4] Sung H.P., Ami P., Joo Y K, Jeong H K, And Suk B K; (2009) J Gynecol Oncol. A Case Of Non-Gestational Choriocarcinoma Arising In The Ovary Of A Postmenopausal Woman; 20(3): 192-194.

[5] Elvira B., Cringua.I., C T.V., Monica M.C., Costin B., (2015) Roman Journal Of Morphological Embryology.Gestational Choriocarcinoma After Term Pregnancy: A Case Report;56(1);267-271.

[6] Anthony T.,(1998) British Medical Journal, Unusual Case Of Choriocarcinoma Occurring 12 Months After Delivery - Lesson Of The Week, 316:532. 